01

\title{
Допплеровское перераспределение по частотам при когерентном излучении фотонов атомами в оптически плотной среде
}

\author{
(C) М.Н. Арасланова, Н.И. Косарев ", М.С. Эльберг
}

Сибирский фредеральный университет, Институт цветных металлов и материаловедения, 660025 Красноярск, Россия

؟ e-mail: kosarev_nikolai@mail.ru

Поступила в редакцию 06.03.2018 г.

\begin{abstract}
Численно решена задача о переносе линейчатого излучения в парах натрия при фотовозбуждении лазерным излучением резонансной линии с длиной волны $\lambda=589.6 \mathrm{~nm}$. Влияние допплеровских сдвигов частоты на когерентное в системе отсчета атома излучение фотонов при формировании контура эмиссионной линии учитывалось, используя модель частичного перераспределения по частотам. Для малой оптической толщины среды $\tau_{0} \leq 1$ допплеровское перераспределение по частотам в лабораторной системе отсчета дает более высокие значения интенсивности в ядре эмиссионного контура по сравнению с моделью полного частотного перераспределения. Для оптически плотной среды тепловое движение атомов приводит к усилению реабсорбции в ядре линии излучения, а в крыльях линии интенсивность усиливается по сравнению с моделью полного перераспределения. Зависимость фактора пленения Холстейна от оптической толщины ближе к аналитической зависимости, чем модель полного перераспределения. Это объясняется тем, что частоты излученных фотонов в лабораторной системе отсчета попадают в ядро спектрального контура, что усиливает эффекты резонансного пленения излучения в плотной среде.
\end{abstract}

DOI: $10.21883 /$ OS.2018.11.46813.68-18

\section{Введение}

Атом при поглощении резонансного фотона переходит в возбужденное состояние, а затем при радиационном распаде излучает фотон. При использовании модели полного перераспределения по частотам (ППЧ) излученный фотон совершенно не „помнит “ частоту, на которой он был поглощен. Предположение о ППЧ оправдано для сред с большой оптической толщиной, для которых фотон, прежде чем он покинет среду, испытает многочисленные акты переизлучения. В результате такого „блуждания“ излученного фотона действительно можно предположить потерю им памяти о частоте поглощенного фотона. Предположение о ППЧ в процессе формирования спектров свечения оптически плотных сред является приближенным, так же как и предположение о полностью когерентном рассеянии фотонов атомами. Более строгое описание процесса излучения фотонов атомами описывается функцией перераспределения фотонов по углам и частотам, учитывающей характер уширения основного и возбужденного уровней резонансной линии атома [1]. Актуальность численного исследования задачи о формировании эмиссионных спектров свечения газовых сред при частичном перераспределении по частотам (ЧПЧ) вызвана предположением о возможном проявлении данных эффектов в условиях эксперимента [2].

В работах [3-6] исследовалось влияние различных функций перераспределения фотонов по частотам на формирование контура спектральной линии и на эффективное время высвечивания оптически плотных сред. Однако неисследованным в [3-6] остался случай полностью когерентного в системе отсчета атома излучения фотонов. В лабораторной системе отсчета каждый атом имеет тепловую скорость, и следовательно благодаря допплеровскому сдвигу частоты поглощенного и излученного фотонов будут отличаться. Следовательно, если в системе отсчета атома излучение полностью когерентно, то для покоящегося относительно лабораторной системы наблюдателя спектральная линия будет формироваться ансамблем движущихся атомов. Такой случай полезно рассмотреть для исследования эффектов допплеровского перераспределения при формировании спектральной линии и сравнения полученных результатов с моделью ППЧ. Кроме того, поскольку исследуется перенос линейчатого излучения, то крылья линии не будут залиты континуумом. Следовательно, эффекты полной когерентности при излучении фотонов в системе отсчета атома, вероятно, будут по-разному проявляться для ядра линии и ее крыльев.

В настоящей работе численно решена задача о фотовозбуждении резонансным лазерным излучением паров натрия при учете переноса излучения в цилиндрическом объеме и ЧПЧ. Исследуется влияние эффектов частичного перераспределения на формирование контура спектральной линии атомов натрия с длиной волны $\lambda=589.6 \mathrm{~nm}$. и на холстейновское [7] эффективное время высвечивания паров. Процесс излучения света атомами натрия моделировался усредненной по углам 
функцией перераспределения $R_{I}[1]$, учитывающей допплеровское перераспределение частот для покоящегося наблюдателя при чисто когерентном рассеянии фотонов в системе отсчета атома.

\section{Скоростные уравнения баланса населенностей двухуровневых атомов при частичном перераспределении по частотам}

Предположим, что лазерным излучением возбуждается резонансная линия атома натрия $3 S_{1 / 2} \leftrightarrow 3 P_{1 / 2}$ с длиной волны $\lambda=589.6 \mathrm{~nm}$. Пары атомов натрия находятся в испаренном состоянии в кювете цилиндрической формы. Когерентными процессами пренебрежем ввиду малой интенсивности лазерного излучения и значительно большей его ширины по сравнению с допплеровской шириной линии. Следовательно, фотовозбуждение атомов будет происходить однородно в пределах всей линии поглощения. При сделанных предположениях кинетика фотовозбуждения двухуровневых атомов может быть описана скоростными уравнениями баланса населенностей. Их использование оправдано, если частотная ширина лазерного излучения превышает частоту Раби $\Omega_{\mathrm{p}} \approx 10^{8} \sqrt{I_{0}}$, где $I_{0}\left[\mathrm{~W} / \mathrm{cm}^{2}\right]$ есть интегральная по частоте интенсивность лазерного излучения [8]. Для параметров численной модели интенсивность лазерного излучения $I_{0}$ не превышала значений $\sim 1 \mathrm{~W} / \mathrm{cm}^{2}$, а допплеровская ширина линии $\Delta v_{\mathrm{D}} \approx 10^{10} \mathrm{~Hz}$. При таких значениях частотная ширина лазерного излучения $\Delta v_{\mathrm{L}}=4 \Delta v_{\mathrm{D}}$ более чем на порядок превышала частоту Раби, что полностью оправдывает использование уравнений баланса населенностей.

Процесс переизлучения фотонов атомами будем описывать усредненной по углам функцией перераспределения $R\left(v^{\prime}, v\right)$. Произведение $R\left(v^{\prime}, v\right) d v^{\prime} d v$ представляет собой совместную вероятность излучения фотона, имевшего в лабораторной системе отсчета частоту в интервале $\left(v^{\prime}, v^{\prime}+\Delta v\right)$, в частотный интервал $(v, v+\Delta v)$. В соответствии с работами [3-6] через $N_{1}$ обозначим населенность основного состояния резонансной линии. Возбужденное состояние разобьем на подуровни с населенностями $N_{2}(v)$, соответствующие различным частотам излученных фотонов. Эти населенности определяют долю возбужденных атомов, которые при спонтанном распаде излучат фотоны на частоте $v$. Полная населенность возбужденного уровня $N_{2}$ получится, если проинтегрировать населенности возбужденных подуровней по частотам,

$$
N_{2}=\int_{0}^{\infty} N_{2}(v) d v
$$

Введем вспомогательную переменную $(v, v+\Delta v) n_{2}$, используя соотношение

$$
N_{2}(v)=n_{2}(v) \Phi(v)
$$

в котором функция $\Phi(v)$ дает частотное распределение атомов по подуровням возбужденного состояния. При естественном фотовозбуждении $\Phi(v)$ представляет собой контур линии поглощения [1]. Соотношение (1) выделяет из $N_{2}(v)$ явную зависимость населенностей подуровней возбужденного состояния от частоты $v$. Теперь $n_{2}(v)$ будет зависеть от $v$ неявно. Для населенностей $N_{2}(v)$ подуровней верхних состояний запишем скоростное уравнение баланса в следующем виде:

$$
\begin{aligned}
d N_{2}(v) / d t= & -N_{2}(v)\left(A_{21}+B_{21} J_{v}\right) \\
& +N_{1} B_{12} \int_{0}^{\infty} R\left(v^{\prime}, v\right) J_{v^{\prime}}, d v^{\prime}
\end{aligned}
$$

с начальным условием

$$
\left.N_{2}(v)\right|_{t=0}=0,
$$

предполагая, что в момент времени $t=0$ не было атомов в возбужденном состоянии. Первое и второе слагаемые в правой части выражения (2) представляют собой соответственно скорости спонтанного распада атомов с подуровня $v$ и вынужденного фототушения. Величины $J_{v^{\prime}}$ и $J_{v}$ - интегральные по углам на частоте $v^{\prime}$ и $v$ интенсивности, формируемые в среде под действием внутренних источников фотонов и внешним излучением. Последнее слагаемое в (2) отвечает за скорость прихода на подуровень $v$ с основного состояния, в котором $R\left(v^{\prime}, v\right)$ - функция перераспределения фотонов по частотам. Физический смысл написанного интеграла в уравнении (2) состоит в следующем: количество фотовозбуждений определяется числом актов поглощения фотонов $N_{1} B_{12} J_{v^{\prime}}$ на частоте $v^{\prime}$, которое затем нужно умножить на совместную вероятность $R\left(v^{\prime}, v\right)$ поглощения фотона на частоте $v^{\prime}$ и излучения на частоте $v$. Затем это произведение следует проинтегрировать по частотам $v^{\prime}$ всех поглощенных фотонов.

Скоростное уравнение баланса для заселенности основного состояния имеет вид

$$
d N_{1} / d t=-N_{1} B_{12} J+N_{2}\left(A_{21}+B_{21} J\right) .
$$

Начальное условия для него $\left.N_{1}\right|_{t=0}=N_{0}$ предполагает, что при $t=0$ концентрация атомов в основном состоянии составляет $N_{0}$. Слагаемое $N_{1} B_{12} J$ в уравнении (3) представляет собой скорость ухода атомов из основного на все подуровни возбужденного состояния под действием фотовозбуждения. Второе и третье слагаемые в (3) соответствуют скоростям заселения основного уровня под действием процессов спонтанного распада 
и вынужденного фототушения. Коэффициенты $J_{v}$ и $J$ в уравнениях (2), (3) определяются из выражений

$$
J_{v}=\int_{0}^{2 \pi} d \varphi \int_{0}^{\pi} \sin (\vartheta) I(\mathbf{r}, \vartheta, \varphi, \nu, t) d \vartheta, \quad J=\int_{0}^{\infty} \Phi_{\nu} J_{\nu} d \nu,
$$

где $\Phi_{v}-$ контур линии поглощения, а $I(\mathbf{r}, \vartheta, \varphi, v, t)-$ интенсивность излучения в точке среды $\mathbf{r}$ в направлении углов $\vartheta$ и $\varphi$ на частоте $v$ в момент времени $t$. Зависимость интенсивности от пространственной и временной переменной определяет неявную зависимость от этих переменных и населенностей уровней $N_{1}, N_{2}$. Их запись от переменных $\mathbf{r}$ и $t$ в уравнениях баланса (2) и (3) была опущена для краткости.

Для стационарного уравнения переноса, учитывая уравнение (1), можем написать

$$
d I / d s=(h v / 4 \pi)\left[-N_{1} B_{12} \Phi_{v} I+n_{2}(v) \Phi_{v}\left(A_{21}+B_{21} I\right)\right],
$$

из которого получаем

$$
d I / d s=\chi_{v}\left[S_{v}-I\right]
$$

где $S_{v}=\eta_{v} / \chi_{v}-$ функция источников, а для коэффициента излучения $\eta_{v}$ и поглощения $\chi_{v}$ имеем

$$
\begin{gathered}
\eta_{v}=\left(2 h v^{3} / c^{2}\right) \alpha_{12}\left(g_{1} / g_{2}\right) n_{2}(v) \Phi_{v}, \\
\chi_{v}=\alpha_{12}\left[N_{1}-\left(g_{1} / g_{2}\right) n_{2}(v)\right] \Phi_{v},
\end{gathered}
$$

где $\alpha_{12}=B_{12} h v_{0} / 4 \pi$, а $g_{1}$ и $g_{2}-$ статистические веса уровней 1 и 2.

Лазерное излучение имело симметричное распределение интенсивности в поперечном сечении и облучало левый торец цилиндра, имеющего высоту $H_{0}$ и радиус $R_{0}$. Поэтому граничное условие для (5) на левой границе $\left(Z=-H_{0} / 2\right)$ для входящего излучения следующее:

$$
I\left(r\left(Z=-H_{0} / 2\right), \theta, \varphi, v, t\right)=\left\{\begin{array}{l}
0, \text { если } \theta \neq 0, \\
I_{L}(v, t), \text { если } \theta=0 .
\end{array}\right.
$$

На правой границе цилиндра $\left(Z=H_{0} / 2\right)$ излучения нет. Тогда граничное условие для нее следующее:

$$
I\left(r\left(Z=H_{0} / 2\right), \theta, \varphi, v, t\right)=0 \text { для } \theta \geq 90^{\circ},
$$

где переменная $\theta$ представляет собой угол между направлением рассеянного фотона и осью симметрии цилиндра $Z$.

Частотно-временная форма лазерного импульса задавалась выражением

$$
I_{L}(v, t)=I_{0} \frac{\left(\Delta v_{L} / 2\right)^{2}}{\left(v-v_{L}\right)^{2}+\left(\Delta v_{L} / 2\right)^{2}}(1-\exp (-t)) .
$$

Здесь $I_{0}-$ максимальная интенсивность импульса, $v_{L}$ - центральная частота лазерного излучения, которая настраивается в центр соответствующей линии атома натрия, а $\Delta v_{L}$ - частотная ширина лазерного излучения, имеющего лоренцовскую зависимость от частоты.

Несмотря на то, что в системе атома переизлучение фотонов когерентное, форма контура линии поглощения $\Phi_{v}$ будет учитывать совместное влияние лоренцовского и допплеровского механизмов уширения. Это нужно для сравнения контуров линии, полученных для моделей полного и частичного перераспределения по частотам между собой для различных оптических толщин среды. Поэтому в нашем случае контур линии поглощения будет описываться нормированной функцией Фойгта:

$$
H(a, x)=\frac{a}{\pi^{3 / 2}} \int_{-\infty}^{\infty} \frac{e^{-y^{2}} d y}{(x+y)^{2}+a^{2}}
$$

где введено обозначение $x=\frac{v-v_{0}}{\Delta v_{L}}$, а параметр Фойгта $a=\frac{\Delta v_{L}}{\Delta v_{D}}$ представляет собой отношение лоренцовской ширины $\Delta v_{L}$ к допплеровской $\Delta v_{D}$. Лоренцовская ширина формируется радиационным и ударным механизмами уширения.

Численные методы решения систем интегродифференциальных уравнений вида (1)-(9) описаны в работе [9]. Эти методы были использованы для расчета эмиссионных контуров линии свечения в работах [3-6] для других моделей перераспределения фотонов по частотам. Разработанные в [9] алгоритмы были адаптированы для учета полностью когерентного излучения фотонов атомами. Время $t$ в выражении (8) измерялось в наносекундах, и все построенные графики соответствовали длительным временам воздействия лазерным излучением $(t=50 \mathrm{~ns})$. На таких временах интенсивность лазерного излучения уже выходит на стационарное значение, и процесс фотовозбуждения атомов натрия лазерным излучением можно считать установившимся во времени.

\section{Модель частичного перераспределения фотонов по частотам}

Функция перераспределения $R\left(v^{\prime}, v\right)$, соответствующая когерентному в системе отсчета атома излучению фотона, задается [1] формулой

$$
R_{1}\left(x^{\prime}, x\right)=\pi^{-1 / 2} \int_{u_{\min }}^{\infty} \exp \left(-u^{2}\right) d u
$$

в которой $u_{\min }=\max \left(\left|x^{\prime}\right|,|x|\right)$, а $x^{\prime}, x$ - частоты поглощенного и излученного фотонов в допплеровских единицах. Такая модель ЧПЧ является достаточно идеализированной, поскольку предполагает резонансную линию с бесконечно тонкими уровнями энергии. Полностью противоположная данной модели - модель ППЧ. При формировании эмиссионных спектров в реальных газовых средах ни одно из этих предположений в 


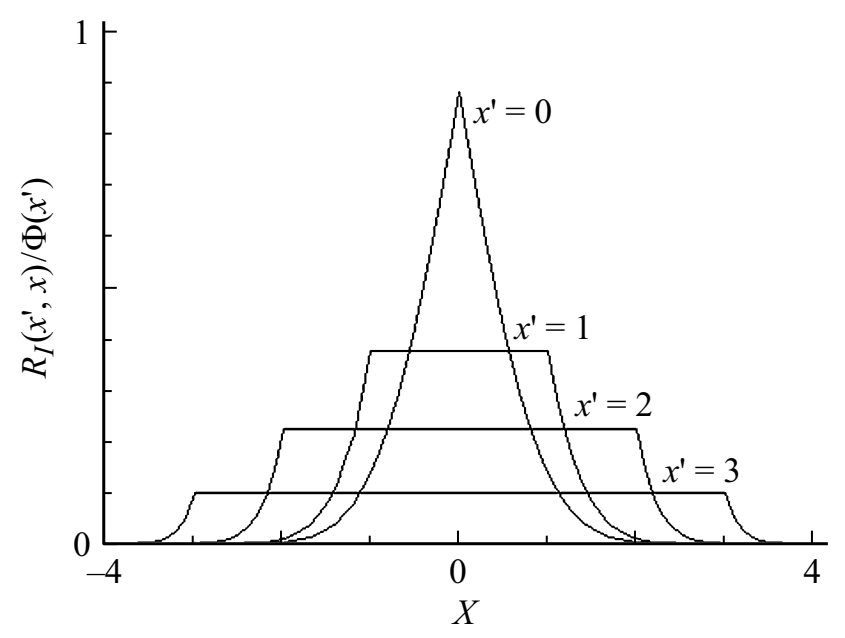

Рис. 1. Вероятность излучения фотона с частотой $x$ при поглощении фотона на частоте $x^{\prime}$ для функции перераспределения $R_{\mathrm{I}}$. По оси абсцисс отложена безразмерная частота $X=\left(v-v_{0}\right) / \Delta v_{D}$ в допплеровских ширинах $\Delta v_{D}$.

точности не выполняется, а реализуется физическая ситуация, которая должна описываться комбинациями функций перераспределения, как в работах [5,6]. Модели перераспределения $R_{I}$ и $R_{\mathrm{CFR}}$ - это на самом деле два предельных гипотетических случая. Первая описывает достаточно разреженный газ с низкой концентрацией нормальных атомов, для которой оптическая толщина среды $\tau_{0} \leq 1$. Вторая - модель ППЧ, наоборот, соответствует газу с высокой концентрацией атомов, такой, что $\tau_{0} \gg 1$.

Целью настоящей работы является исследование эффектов допплеровского перераспределения в процессе формирования эмиссионного спектра, который будет фиксировать внешний наблюдатель в лабораторной системе отсчета. Тепловое движение ансамбля движущихся атомов перемешивает частоты излученных фотонов, и когерентность в атомной системе отсчета должна, естественно, нарушаться. Если бы это была не так, то нужно было бы предположить, что тепловая скорость всех атомов газа равна нулю. Отметим, что для случая полностью когерентного рассеяния в лабораторной системе отсчета нужно было бы в описанную выше модель подставить функцию перераспределения

$$
R\left(x^{\prime}, x\right)=\Phi\left(x^{\prime}\right) \delta\left(x-x^{\prime}\right)
$$

где $\delta$ - дельта-функция. Случай ППЧ будет реализован, если для функции перераспределения использовать выражение $R_{\mathrm{CFR}}=\Phi\left(x^{\prime}\right) \Phi(x)$.

На рис. 1 показаны графики функции $R_{1}\left(x^{\prime}, x\right) / \Phi\left(x^{\prime}\right)$, рассчитанные численно по методу Симпсона [10,11]. Подписи к кривым указывают частоты поглощенных фотонов $x^{\prime}$ для различных значений последнего. Вид графиков показывает, что при поглощении фотона на частоте $x^{\prime}$ излученный фотон с одинаковой вероятностью будет иметь частоту в диапазоне $-\left|x^{\prime}\right| \leq x \leq|x|$.
Вероятность излучения фотона с частотой вне пределов данного диапазона уменьшается экспоненциально с ростом $x$.

\section{Результаты численного моделирования}

\section{Формирование контура спектральной линии}

Рассчитывались кинетика фотовозбуждения и эмиссионный спектр интенсивности флуоресценции в различных лучевых направлениях для паров атомарного натрия на резонансном переходе с длиной волны $\lambda=589.6 \mathrm{~nm}$ при поглощении атомами лазерного излучения. Центральная частота лазерного излучения настраивалась в центр резонансной линии натрия. Варьировались параметры среды и лазерного излучения. Интенсивность лазерного излучения подбиралась значительно меньше интенсивности, насыщающей переход в атоме натрия [12] для того, чтобы в уравнениях баланса (2), (3) превалировали процессы вынужденного фотовозбуждения линии лазерным излучением. При этом лазерное излучение будет в значительной степени поглощаться на частотах резонансной линии и высока роль функции источников, отвечающей в уравнении переноса (5) за интенсивность вторичного излучения. Концентрация атомов натрия определяется давлением насыщенных паров при определенной температуре. Холстейновская оптическая толщина паров $\tau_{0}$ [7] рассчитывалась для центральной частоты линии по радиусу цилиндра для начального момента времени, когда в уравнении (6) все значения $n_{2}(v)=0$. Тогда с учетом нормировки функции $\Phi(x)$ в уравнении (6) и перехода в ней от переменной $v$ к относительным частотам $x$ оптическая толщина $\tau_{0}=\alpha_{12} N_{1} /\left(\Delta v_{D} \sqrt{\pi}\right) R_{0}$. В данном разделе работы приводятся результаты расчетов для цилиндра с отношением высоты $H_{0}$ к диаметру основания $D_{0}=2 R_{0}$, равным единице.

Перенос излучения в оптически плотных газовых средах приводит к неоднородному по объему фотовозбуждению атомов. Их концентрация больше на облученной стороне среды, чем в области геометрической тени [12-15]. Это вызвано сильным поглощением лазерного излучения ненасыщающей интенсивности при его распространении в плотной атомарной среде. Кроме этого, концентрация возбужденных атомов всегда выше внутри газового объема, чем у боковой его поверхности, где выход фотонов из среды приводит к распаду возбуждения. В условиях неоднородного фотовозбуждения атомов интенсивность эмиссии по заданной оптической трассе формируется в условиях конкуренции поглощения и излучения фотонов. Эмиссионный контур линии излучения для модели ППЧ и допплеровского контура линии поглощения исследован в работах [12-15]. Показано, что вид частотной формы эмиссионного спектра зависит от направления выхода излучения из среды. При рассеянии в направлениях, противоположных внешнему излучению, контур линии не реабсорбирован. Это 

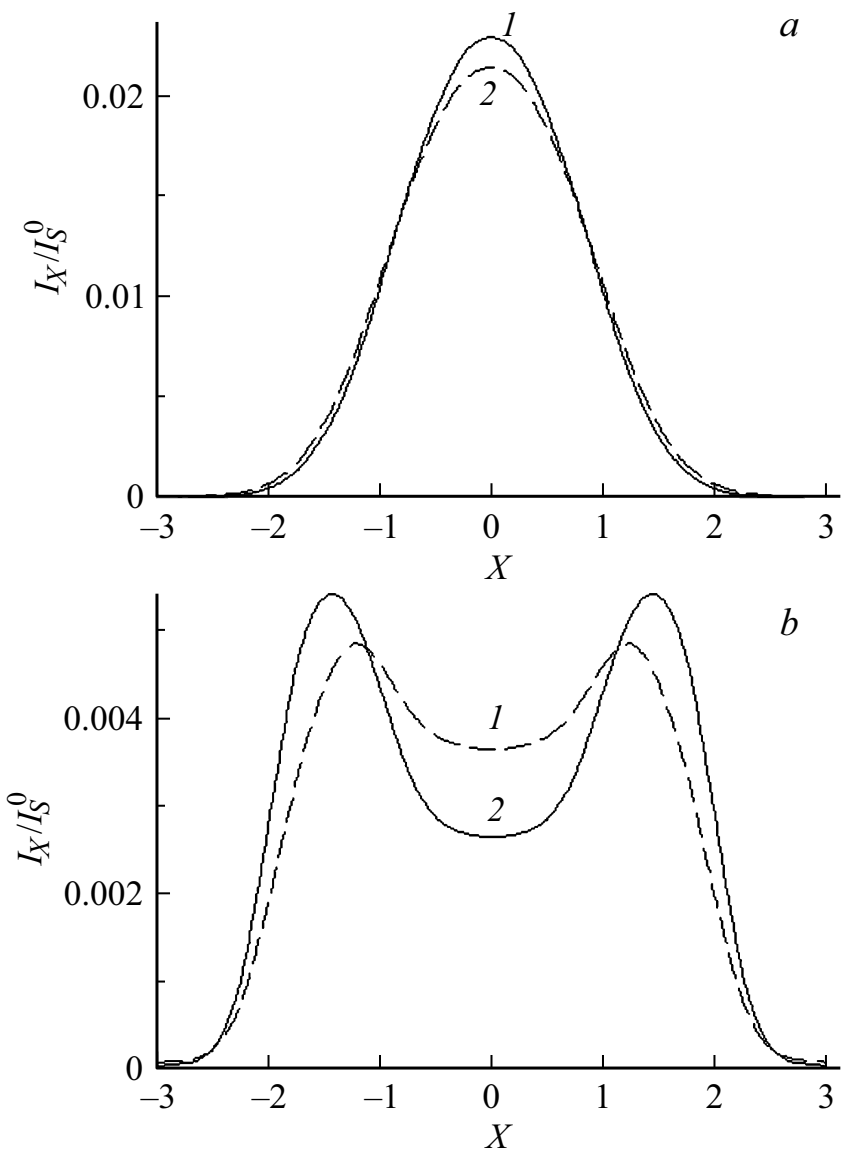

Рис. 2. Частотный контур эмиссионной линии для излучения, рассеянного с боковой границы газовой кюветы, для оптической толщины $\tau_{0}=0.5, I_{0}=10^{-3} \mathrm{~W} / \mathrm{cm}^{2}(a)$ и $\tau_{0}=10$, $I_{0}=2 \cdot 10^{-1} \mathrm{~W} / \mathrm{cm}^{2}(b)$. Сплошные кривые $1-$ модель ЧПЧ, штриховые кривые $2-$ модель ППЧ. Среда имеет форму цилиндра с отношением $H_{0} / 2 R_{0}=1$.

объясняется тем, что такие лучевые трассы соответствуют рассеянию фотонов в направлении уменьшения коэффициента поглощения. При выходе фотонов из области геометрической тени контур спектральной линии оказывается реабсорбированным - в этом случае фотоны распространяются в направлении увеличения коэффициента поглощения.

Исследуем важный с практической точки зрения случай рассеяния излучения в направлении, перпендикулярном газовой кювете с середины боковой поверхности. На рис. 2 показаны частотные контуры эмиссионной линии, формируемой по оптической трассе, проходящей через центр цилиндра для различных значений $\tau_{0}$, соответствующих разным начальным концентрациям атомов $N_{0}$. Сплошные кривые 1 соответствуют модели ЧПЧ, штриховые кривые 2 - случаю ППЧ, когда в модели используется функция $R_{\mathrm{CFR}}$.

Для малой оптической толщины $\tau_{0}=0.5$ контур эмиссионной линии для случая частичного перераспределения имеет вид колоколообразной кривой с максимумом в центре линии (кривая 1, рис. 2,a). Форма линии для модели ППЧ (кривая 2) отличается от кривой 1 более низкой интенсивностью в ядре контура на частотах $-1<x<1$. Для крыльев линии модель ППЧ дает небольшое завышение интенсивности по сравнению с моделью ЧПЧ. Еще раз отметим, что функция перераспределения $R_{\mathrm{I}}$ соответствует достаточно разреженному газу, в котором возбужденное состояние резонансной линии не уширено никакими механизмами. При этом допплеровское перераспределение по частотам приводит к тому, что фотоны, поглощенные на частоте $x^{\prime}$, переизлучаются атомами с одинаковой вероятностью в диапазоне частот $-\left|x^{\prime}\right|<x<\left|x^{\prime}\right|$. Из рис. 1 видно, что при частоте фотона $x^{\prime}=1$ вероятность излучения фотона в диапазоне $-1<x<1$ равна $\approx 0.37$. Если частота $x^{\prime}$ выходит за рамки данного диапазона, то вероятность излучения на частоте $x$ становится еще меньше. Но в оптически тонкой среде эффекты перепоглощения фотонов нормальными атомами незначительны. Следовательно, когерентность в системе отсчета атома для малой оптической толщины оставляет излученные фотоны в частотном диапазоне $1<x<-1$, для которых коэффициент излучения достаточно высок. Эти фотоны, излученные в ядре линии, усиливают центральные частоты эмиссионного контура. Распределение интенсивности по частоте вне диапазона $1<x<-1$ уже достаточно хорошо описывается моделью ППЧ: кривые 1 и 2 на рис. 2 почти совпадают. Следовательно, когерентность в системе отсчета атома при допплеровском перераспределении фотонов в системе отсчета наблюдателя приводит к усилению интенсивности линии в ядре контура для малой оптической толщины среды $\tau_{0} \leq 1$.

Для оптической толщины $\tau_{0}=10$ наблюдаемая картина в распределении интенсивности эмиссии по частоте (рис. 2,b) полностью противоположна предыдущему случаю. Во-первых, полная ширина на половине максимума интенсивности (FWHM) контура эмиссионной линии больше FWHM эмиссионного контура для оптически тонкого случая. Это объясняется тем, что величина FWHM зависит от температуры атомных паров, которая и определяет концентрацию нормальных атомов в газовой кювете. Поэтому чем больше оптическая толщина, тем выше температура паров и, следовательно, больше FWHM. Во-вторых, появляется эффект реабсорбции спектрального контура. Но теперь в ядре допплеровского контура модель ЧПЧ (кривая 1) дает более низкие значения интенсивности по сравнению с моделью ППЧ (кривая 2) для частотного диапазона излученных фотонов $|x| \leq 1.2$. Следовательно, когерентное рассеяние фотонов усиливает явление реабсорбции в плотной среде. Одновременно с реабсорбцией ядра контура происходит усиление интенсивности в близкой к ядру линии области $|x|>1.2$, где коэффициент поглощения уменьшается, и роль процессов пленения снижается. В крыле частотного контура на частотах 
$|x|>2.4$ коэффициент поглощения уменьшается настолько, что интенсивность спадает до нуля как для ЧПЧ-, так и для ППЧ-моделей. Описанные эффекты уширения линии и одновременно усиления реабсорбции центральной области эмиссионной контура проявляются еще сильнее с ростом оптической толщины $\tau_{0}$. Такое поведение контура эмиссионной линии вызвано совместным влиянием допплеровского сдвига частоты когерентных фотонов и большой оптической толщины среды.

\section{Эффективное время высвечивания паров натрия}

В оптически плотных средах излучение является запертым в газовом объеме за счет пленения и существует в форме возбужденных атомов. Для исследования времени жизни возбужденного состояния резонансной линии Холстейном введено понятие эффективного времени высвечивания среды [7]. При этом неважно, как создаются возбужденные атомы. Если накачать среду резонансным лазерным излучением большой интенсивности и малой длительности, сравнимой или меньшей естественного времени жизни возбужденного уровня атома натрия $\tau_{\text {nat }}=1 / A_{21}=1.75 \cdot 10^{-8} \mathrm{~s}$, то распад населенностей в условиях пленения фотонов будет протекать значительно дольше, чем $\tau_{\text {nat }}$ в режиме послесвечения. Поэтому в начальный момент времени можно задавать распределение населенностей атомов по состояниям, соответствующее случаю сильного насыщения среды на резонансном переходе. Эти населенности определяются через статистические веса уровней:

$$
N_{2} \approx g_{2} /\left(g_{1}+g_{2}\right) N_{0}, \quad N_{1} \approx g_{1} /\left(g_{1}+g_{2}\right) N_{0} .
$$

Для рассматриваемой линии в атоме натрия $\left(g_{2}=4\right.$, $\left.g_{1}=2\right)$ для $N_{2}$ и $N_{1}$ получим значения $N_{2} \approx 0.66 N_{0}$, $N_{1} \approx 0.34 N_{0}$. Эффективное время высвечивания $\tau_{\text {eff }}$ рассчитывалось по декременту уменьшения во времени интегральной в линии интенсивности послесвечения по такой же оптической трассе, что и эмиссионный контур линии излучения. Такой способ нахождения величины $\tau_{\text {eff }}$ основан на предположении, что уменьшение интенсивности излучения во времени, как и распад населенностей, носит одноэкспоненциальный характер [7] $J(t) \approx \exp \left(-t / \tau_{\text {eff }}\right)$.

Эффективное время высвечивания $\tau_{\text {eff }}$ по Холстейну для модели ППЧ и допплеровского контура линии поглощения определяется [7] выражением

$$
\tau_{\mathrm{eff}}=\frac{1}{g_{D} A_{21}} \tau_{0} \sqrt{\pi \ln \left(\tau_{0}\right)},
$$

в котором геометрический фактор $g_{D}=1.6$ для бесконечно длинного цилиндра и $g_{D}=0.94$ для плоского слоя. Приближение (11) справедливо для $\tau_{0} \geq 7$. В отличие от приближенной модели Холстейна численная модель (1)-(10) не зависит от геометрического

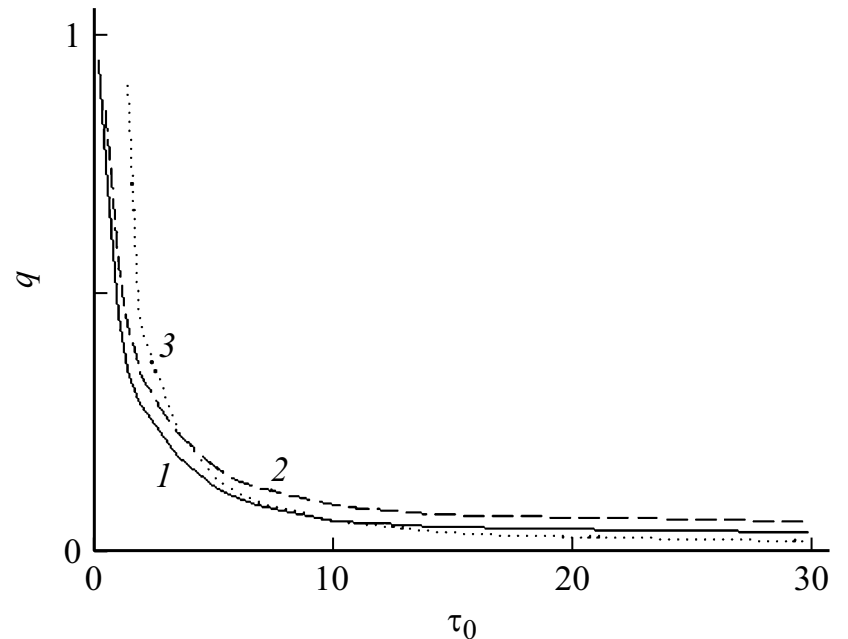

Рис. 3. Зависимости фактора пленения $q$ от оптической толщины $\tau_{0}$ паров натрия для цилиндра с отношением $H_{0} / 2 R_{0}=6$. Сплошная кривая $1-$ модель ЧПЧ, штриховая кривая 2 - ППЧ, пунктирная кривая 3 - теория Холстейна для бесконечно длинного цилиндра.

фактора, а контур линии поглощения учитывает совместное влияние допплеровского и столкновительного механизмов уширения линии поглощения атома. Кроме того, эффективное время высвечивания $\tau_{\text {eff }}$ рассчитывается по интегральному в линии излучению в широком диапазоне оптических толщин $\tau_{0}$, задавая высоту $H_{0}$, радиус $R_{0}$ цилиндра и начальную концентрацию $N_{0}$ атомов.

Вместо эффективного времени высвечивания рассчитывался фактор пленения Холстейна $q$, который представляет собой отношение естественного времени жизни возбужденного состояния к эффективному времени $q=\tau_{\text {nat }} / \tau_{\text {eff. }}$ По такому определению в асимптотической модели Холстейна фактор пленения $q$ не зависит от химического элемента, а определяется только геометрической конфигурацией газового объема и типом уширения линии.

На рис. 3 показаны зависимости от оптической толщины $\tau_{0}$ среды фактора пленения $q$ для цилиндров с отношением $H_{0} / 2 R_{0}=6$. В работах [3-6] численное исследование фактора пленения Холстейна показало, что для цилиндров с отношением $H_{0} / 2 R_{0} \geq 6$ нет заметного отличия в поведении зависимости $q$ от $\tau_{0}$. Поэтому для таких цилиндров выход излучения из объема осуществляется через боковую поверхность и они, с точки зрения процессов пленения фотонов, эквивалентны бесконечно длинному цилиндру в модели Холстейна [7]. Кривая 1 на рис. 3 рассчитана для ЧПЧ, а кривая 2 соответствует модели ППЧ. Кривая 3 отображает зависимость $q$ от $\tau_{0}$, рассчитанную по модели Холстейна для бесконечно длинного цилиндра (11). Оптическая толщина паров увеличивается с ростом начальной концентрации атомов натрия. При этом усиливаются и 
эффекты пленения резонансного излучения. Это, естественно, приводит к увеличению эффективного времени высвечивания паров и, следовательно, к уменьшению $q$ (кривая 1).

Из сравнения кривых 1 и 2 видно, что модель ЧПЧ дает заниженное значение фактора $q$ по сравнению с моделью ППЧ. Это вызвано тем обстоятельством, что функция перераспределения $R_{\mathrm{I}}$ отвечает за когерентное излучение фотонов в системе отсчета атома. При этом в лабораторной системе отсчета излученные фотоны остаются в ядре эмиссионного контура линии. Для модели ППЧ вероятность излученных фотонов не зависит от частоты поглощенных. Поэтому фотоны, поглощенные в ядре линии, могут быть переизлучены и на частотах, удаленных от $v_{0}$, где меньше коэффициент поглощения. Следовательно, для модели ЧПЧ эффекты самопоглощения сильней, а эффективное время высвечивания больше, чем для ППЧ. Более того, сравнение кривых 1 и 3 показывает, что расчетная зависимость для модели ЧПЧ ближе к кривой Холстейна для оптических толщин $\tau_{0} \geq 7$, для которых справедлива асимптотическая формула (11). Но все-таки, расчетная кривая 1 дает более завышенное значение для фактора $q$, чем кривая Холстейна. Это объясняется тем, что в численной модели использован фойгтовский контур линии, а в модели Холстейна - допплеровский. Последний хорошо описывает ядро и плохо крылья спектральной линии, где осуществляется радиационный перенос в оптически плотных средах. Поэтому допплеровский контур линии должен давать завышенное значение для эффективного времени высвечивания по сравнению с фойгтовским контуром.

\section{Основные результаты и выводы}

Построена модель переноса излучения в оптически плотных парах атомарного натрия при лазерном фотовозбуждении резонансной линии на переходе с длиной волны $\lambda=589.6 \mathrm{~nm}$. Численный алгоритм решения системы интегро-дифференциальных уравнений построен для цилиндрической геометрии среды и функции перераспределения $R_{\mathrm{I}}$, учитывающей допплеровское перераспределение по частотам когерентно излученных атомами фотонов.

Рассчитаны кинетика фотовозбуждения резонансной линии натрия и эмиссионный спектр свечения паров на длине волны $\lambda=589.6 \mathrm{~nm}$. Интенсивность флуоресценции формируется в условиях конкуренции процессов поглощения и излучения фотонов. Форма эмиссионного контура имеет самообращенный вид при рассеянии с боковой поверхности газовой кюветы для оптических толщин $\tau_{0}>1$.

Для малых оптических толщин $\tau_{0} \leq 1$ допплеровское перераспределение когерентно излученных фотонов не уширяет спектральную линию значительно по сравнению с ППЧ. Основное влияние когерентности в системе отсчета атома проявляется в усилении интенсивности для ядра эмиссионного спектра на частотах $-1<x<1$ в единицах допплеровской ширины линии. Это связано с тем, что, во-первых, при поглощении фотона на частоте $x^{\prime}$ излученные фотоны с равной вероятностью попадают в частотный интервал $-\left|x^{\prime}\right| \leq x \leq|x|$. Вне этого диапазона вероятность излучения для фотонов уменьшается экспоненциально. Во-вторых, для оптически тонкой среды фотоны, излученные в ядре контура, беспрепятственно покидают среду без заметного поглощения, формируя не реабсорбированный контур линии.

С ростом оптической толщины многократное переизлучение когерентных фотонов усиливает именно роль ядра спектральной линии и увеличивает реабсорбцию эмиссионного контура примерно в том же диапазоне частот $-1.2<x<1.2$. При этом на частотах, близких к ядру, происходит усиление интенсивности и соответственно уширение спектрального контура эмиссионной линии по сравнению с ППЧ.

Усиление реабсорбции для когерентного рассеяния фотонов приводит к замедлению эффективного времени высвечивания среды по сравнению с моделью ППЧ. При этом кривая зависимости фактора пленения от оптической толщины среды ближе к холстейновской зависимости, чем кривая, соответствующая ППЧ. Чтобы оценить точность полученных результатов по влиянию допплеровского перераспределения в лабораторной системе отсчета когерентно излученных фотонов и определить диапазон применимости модели частичного перераспределения по оптическим толщинам среды, нужно было бы провести детальное сравнение эмиссионного контура спектральной линии и эффективного времени высвечивания не только с ППЧ-результатами, но также и с другими моделями рассеяния атомами фотонов, учитывающими естественное и столкновительное уширение возбужденного состояния атома. Подобное исследование является предметом дальнейших изысканий авторов в рамках данной тематики.

\section{Список литературы}

[1] Михалас Д. Звездные атмосферы. Ч. 1,2. М.: Мир, 1982

[2] Romberg A., Kunze H.-J. // JQSRT. 1988. V. 39. N 2. P. 99.

[3] Косарев Н.И. // Опт. и спектр. 2007. Т. 102. № 1. С. 13.

[4] Косарев Н.И. // Опт. и спектр. 2007. Т. 102. № 5. С. 718.

[5] Косарев Н.И. // Опт. и спектр. 2008. Т. 104. № 1. С. 5.

[6] Kosarev N.I. // J. Phys. B . 2008. V. 41. N 22. P. 225401.

[7] Holstein T. // Phys. Rev. 1947. V. 72. P. 1212. Ibid. 1951. V. 83. P. 1159.

[8] Карлов Е.М. Лекции по квантовой электронике. М., 1983. $318 \mathrm{c}$.

[9] Косарев Н.И. // Математическое моделирование. 2008. T. 20. № 3. C. 87.

[10] Арасланова М.Н., Косарев Н.И. // Моделирование неравновесных систем. Материалы XX Всероссийского семинара. Красноярск: Институт вычислительного моделирования СО РАН, 2017. С. 3. 
[11] Арасланова М.Н., Косарев Н.И. // Моделирование неравновесных систем. Материалы XX Всероссийского семинара. Красноярск: Институт вычислительного моделирования СО РАН, 2017. С. 8.

[12] Косарев Н.И. // Опт. и спектр. 2006. Т. 101. № 1. С. 64.

[13] Косарев Н.И., Шкедов И.М. // Оптика атмосферы и океана. 1993. Т. 6. № 10. С. 1298.

[14] Косарев Н.И., Шкедов И.М. // Оптика атмосферы и океана. 1999. Т. 12. № 1. С. 30.

[15] Косарев Н.И., Шкедов И.М. // Оптика атмосферы и океана. 1995. Т. 8. № 12. С. 1752. 